Pure and Applied Mathematics Quarterly

Volume 8, Number 3

$741-754,2012$

\title{
The Model of Paths
}

\author{
Zhixiang $\mathrm{Wu}$
}

\begin{abstract}
In the present paper, we define a new kind of model of paths. Using this new model, we obtain the well-known first Weyl character formula and prove the decomposition theorem of the tensor product of two simple modules over a Kac-Moody algebra.
\end{abstract}

Keywords: Affine Lie algebras, Weyl formula, path model.

\section{INTRODUCTION}

The aim of the present paper is to introduce a new path model. This new path model consists of Littelmann's paths $([5,6])$ and a new kind of operators acting on Littelmann's paths. This newly defined operator is called tail-flip operator. Using the tail-flip operator, one can obtain the first Weyl formula and the tensor product decomposition theorem of two simple modules over a Kac-Moody algebra, without using the theory of $L S$ paths.

To be more precise, let $\alpha$ be a real root of a symmetric "nontwisted" affine Kac-Moody algebra $\mathcal{G}^{\prime}, \pi$ be a piecewise linear path in the space $\mathcal{X}$ spanned over the rational number field by the weights of the affine Kac-Moody algebra $\mathcal{G}^{\prime}$. We use $[0,1]_{\mathbf{Q}}$ to denote the set $\{x \mid x$ is a rational number, $0 \leq x \leq 1\}$. Define a tail-flip operator $T_{\alpha, x}$ for any $x \in[0,1]_{\mathbf{Q}}$ as follows.

Received April 12, 2008.

2000 Mathematics Subject Classification.Primary 17B67, Secondary 17B10.

The author is sponsored by SRF for ROCS, SEM. He is also sponsored by the Scientific Research Foundation of Zhejiang Provincial Education Department. 
If $\left(\pi(x), \alpha^{y}\right)$ is an integer, and either $\left(\pi(t), \alpha^{y}\right) \geq\left(\pi(x), \alpha^{y}\right)$ for $t \geq x$ or $x=0$, then

$$
T_{\alpha, x}(\pi)(t)=\left\{\begin{array}{ll}
\pi(t), & \text { for } 0 \leq t \leq x \\
\pi(x)+s_{\alpha}(\pi(t)-\pi(x)), & \text { for } x \leq t \leq 1
\end{array} .\right.
$$

Otherwise, $T_{\alpha, x}(\pi)(t)=0$ for all $t \in[0,1]$. If $\alpha=\alpha_{i}$ is a simple root, then we usually use $T_{i, x}$ to denote the operator $T_{\alpha_{i}, x}$.

Let $B$ be a set of piecewise linear paths, which is stable under the action of tail-flip operators $T_{i, x}$ for all $i$ and all rational number $x \in[0,1]_{\mathbf{Q}}$. Define Char $B:=\sum_{\eta \in B} e^{\eta(1)}$ formally. We call Char $B$ the character of $B$. For example, let $\mathcal{B}$ be the algebra generated by all operators $T_{i, x}$ over the integer number ring $\mathbf{Z}$, let $\pi=t \lambda, t \in[0,1]_{\mathbf{Q}}$, for some dominant weight $\lambda$. If $B$ is the set of all paths contained in $\mathcal{B} \pi$, then $\operatorname{Char} B$ is the character formula of a simple module with highest weight $\lambda$. This result is proved in Proposition 4.1. Suppose $\rho$ is a weight satisfying $\left(\rho, \alpha_{i}\right)=1$ for all simple roots $\alpha_{i}$. Then the character of $B$ can be computed by using the the Weyl group $\bar{W}$ of the Kac-Moody algebra $\mathcal{G}^{\prime}$ in the following way.

Theorem(1) Let $\Pi_{0}^{+}$be the set of the piecewise linear paths such that Im $\eta$ is in the interior of $\mathcal{C}($ for $t>0)$, where $\mathcal{C}$ is the Tits cone of a nontwised affine Kac-Moody algebra $\mathcal{G}^{\prime}$. Suppose $B$ is a set of piecewise linear paths, which is stable under the action of $T_{i, x}$ for all rational number $x \in[0,1]$ and simple roots $\alpha_{i}$ of $\mathcal{G}^{\prime}$. Then

$$
\left(\sum_{w \in \bar{W}} \operatorname{sgn}(w) e^{w(\rho)}\right) \operatorname{Char} B=\sum_{\eta \in B, \rho \otimes \eta \in \Pi_{0}^{+}}\left(\sum_{w \in \bar{W}} \operatorname{sgn}(w) e^{w(\rho+\eta(1))}\right) .
$$

(2) For any dominant weight $\mu$, let $V_{\mu}$ be the corresponding irreducible $\mathcal{G}^{\prime}$ representation with highest weight $\mu$, then

$$
\operatorname{Char} B=\sum_{\eta \in B, \rho \otimes \eta \in \Pi_{0}^{+}} \operatorname{CharV}_{\eta(1)}
$$

The formula $\left(\sum_{w \in \bar{W}} \operatorname{sgn}(w) e^{w(\rho)}\right)$ Char $B=\sum_{\eta \in B, \rho \otimes \eta \in \Pi_{0}^{+}}\left(\sum_{w \in \bar{W}} \operatorname{sgn}(w)\right.$. $\left.e^{w(\rho+\eta(1))}\right)$ is called the first Weyl character formula. We will prove this theorem by using tail-flip operators. 
Finally, let us give a brief outline of this paper. In Section 2, we review some basic facts related to root systems of a nontwised affine Kac-Moody algebra $\mathcal{G}^{\prime}([3$, p.96]). In Section 3, we define a kind of tail-flip operators on piecewise linear paths without any restriction on the paths and roots. We call this kind of operators absolute tail-flip. We obtain some properties of these operators, and compare these operators with the root operators defined by Littelmann. In Section 4 , we prove the above mentioned theorem.

\section{NotATiOn}

Let $I=\{1, \cdots, l\}$ and $A=\left(a_{i j}\right)_{l \times l}$, where $A$ is the Cartan matrix of some finite dimensional simple Lie algebra $\mathcal{G}$ over the complex field C. Fix a Cartan subalgebra $\mathcal{H} \subseteq \mathcal{G}$. Let $\left\{\alpha_{i}, i=1, \cdots, l\right\}$ ( respectively, $\left\{\alpha_{i}^{\breve{i}}, i=1, \cdots, l\right\}$ ) be the basis of $\mathcal{H}^{*}$ ( respectively, of $\mathcal{H}$, such that $\alpha_{i}\left(\alpha_{j}\right)=a_{i j}$. We also denote $\alpha_{i}\left(\alpha_{j}\right)$ by $\left(\alpha_{j}, \alpha_{i}\right)$. Define the fundamental weight $\omega_{i} \in \mathcal{H}^{*}$ of $\mathcal{G}$ by $\omega_{i}\left(\alpha_{j}^{j}\right)=\delta_{i j}$, where $\delta_{i j}$ is the Kronecker's symbol. Let $P_{0}$ be the free abelian group generated by $\omega_{i}, i=1, \cdots, l$. Let $\theta=\sum_{i=1}^{l} a_{i} \alpha_{i}$ be the highest root of $\mathcal{G}$ with respect to $\mathcal{H}$ and $\theta=\sum_{i=1}^{l} a_{i} \breve{\alpha_{i}}$ the corresponding coroot.

Set $I^{\prime}=I \cup\{0\}$ and let $A^{\prime}=\left(a_{i j}\right)_{i, j \in I^{\prime}}$ be the generalized Cartan matrix of the "nontwisted" affine Lie algebra $\mathcal{G}^{\prime}$ associated with $\mathcal{G}([3, \mathrm{p} .96])$. As a vector space,

$$
\mathcal{G}^{\prime}=\mathcal{G} \otimes_{\mathbf{C}} \mathbf{C}\left[t, t^{-1}\right] \oplus \mathbf{C} c \oplus \mathbf{C} \partial
$$

where $c$ is the canonical central element and $a d \partial=t \frac{d}{d t}$. Then $\mathcal{H}^{\prime}=\mathcal{H} \oplus \mathbf{C} c \oplus \mathbf{C} \partial$

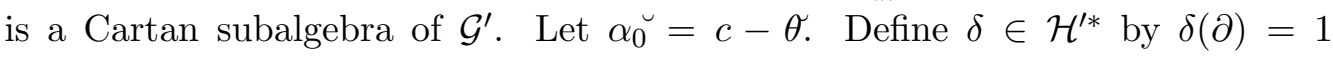
and $\delta(\mathcal{H} \oplus \mathbf{C} c)=0$. Denote $\alpha_{0}=\delta-\theta$. Then $\left\{\alpha_{0}, \alpha_{1}, \cdots, \alpha_{l}\right\}$ (respectively, $\left\{\alpha_{0}, \alpha_{1}, \cdots, \alpha_{l}\right\}$ ) is a set of simple roots of $\mathcal{G}^{\prime}$ (respectively, coroots of $\mathcal{G}^{\prime}$ ). Notice that $\delta\left(\alpha_{i}\right)=\alpha_{i}(c)=0$ for $i=0, \cdots, l$.

Define the fundamental weights $\overline{\omega_{i}} \in \mathcal{H}^{\prime *}$ of $\mathcal{G}^{\prime}$ by $\overline{\omega_{i}}\left(\alpha_{j}\right)=\delta_{i, j}$ and $\overline{\omega_{i}}(\partial)=\delta_{i, 0}$ for $i \in I^{\prime}$. Let $P$ be the free abelian group generated by $\overline{\omega_{i}}, i \in I^{\prime}$. Set $\bar{P}=P \oplus \mathbf{Z} \delta$, where $\mathbf{Z}$ is the ring of integer number. Let $\iota\left(\omega_{i}\right)=\overline{\omega_{i}}-a_{i} \overline{\omega_{0}}$. Then $\iota$ is an embedding map from $P_{0}$ to $P$. Identify $P_{0}$ with its image inside $P$ which in turn coincides with the set $\{\lambda \in P \mid \lambda(c)=0\}$. Let $\xi: \bar{P} \rightarrow \bar{P} / \mathbf{Z} \delta$ be the canonical projection. Notice that $P$ can be identified with $\bar{P} / \mathbf{Z} \delta$ and $\xi\left(\alpha_{0}\right)=-\theta$. 
For all $i \in I^{\prime}$ define an elementary reflection $s_{i} \in A u t \mathcal{H}^{*}$ by $s_{i}(\lambda)=\lambda-$ $\lambda\left(\alpha_{i}{ }^{y} \alpha_{i}\right.$, where $\lambda \in \mathcal{H}^{\prime *}$. The Weyl group $\bar{W}$ of $\mathcal{G}^{\prime}$ (respectively, $W$ of $\mathcal{G}$ ) identifies with the group generated by $s_{i}: i \in I^{\prime}$ ( respectively, $\left.i \in I\right)$. The set of roots of $\mathcal{G}^{\prime}$ is a disjoint union of the set of real roots $\cup_{i \in I^{\prime}} \bar{W} \alpha_{i}$ and imaginary roots $\mathbf{Z} \delta \backslash\{0\}$. We use $\Phi^{+}$to denote the set of all positive roots of $\mathcal{G}^{\prime}$. For any real root $\beta$, let $\beta^{\cup}=\frac{2}{(\beta, \beta)} \beta$ be the corresponding coroot of $\beta$. The reflection $s_{\beta}$ is defined via $s_{\beta}(\lambda)=\lambda-\lambda\left(\beta^{\cup}\right) \beta, \lambda \in \mathcal{H}^{\prime *}$. Observe that $s_{0}=s_{\theta}$ as an automorphism of $P$ and so we can identify $\bar{W}$ with $W$, whenever we consider the action of $\bar{W}$ acting on $P$.

\section{PATHS AND ROOTS}

3.1 Given $a, b \in \mathbf{Q}$ the rational number field, set $[a, b]_{\mathbf{Q}}:=\{x \in \mathbf{Q} \mid a \leq x \leq b\}$. Let $\lambda$ be an integral weight. For $\mu, \nu \in \bar{W} \lambda$ write $\nu \geq \mu$ if there exists a sequence of weights $\nu=\nu_{0}, \nu_{1}, \cdots, \nu_{s}=\mu$ and positive real roots $\beta_{1}, \beta_{2}, \cdots, \beta_{s}$ such that $\nu_{i}=s_{\beta_{i}} \nu_{i-1}$ and $\left(\nu_{i-1}, \beta_{i}\right)<0$ for all $i=1,2, \cdots, s$. Then $\nu>\mu$ means that $\nu \geq \mu$ and $\nu \neq \mu$. If $\nu>\mu$, then define $\operatorname{dist}(\nu, \mu)$ to be the maximal length $s$ of all possible such sequences. Let $a \in[0,1]_{\mathbf{Q}}$ be a rational number. An $a$-chain for $(\mu, \nu)$ is a sequence $\mu=\lambda_{0}>\lambda_{1}>\cdots>\lambda_{s}=\nu$ of weights in $\bar{W} \lambda$ such that either $s=0$ and $\mu=\lambda_{0}=\nu$ or $\lambda_{i}=s_{\beta_{i}}\left(\lambda_{i-1}\right)$ for some positive real roots $\beta_{1}, \cdots, \beta_{s}$, and $\operatorname{dist}\left(\lambda_{i-1}, \lambda_{i}\right)=1$, and $a\left(\lambda_{i-1}, \beta_{i}^{y}\right) \in \mathbf{Z}$ for all $i=1,2, \cdots, s$. For a sequence $\nu_{1}>\nu_{2}>\cdots>\nu_{s}$ of weights in $\bar{W} \lambda$, and a sequence $a_{0}=0<a_{1}<\cdots<a_{s}=1$ of rational numbers in $[0,1]_{\mathbf{Q}}$, define a path as follows:

$$
\pi(t)=\sum_{i=1}^{j-1}\left(a_{i}-a_{i-1}\right) \nu_{i}+\left(t-a_{j-1}\right) \nu_{j},
$$

for $a_{j-1} \leq t \leq a_{j}$. This path is called an $L S$-path if for all $i=1,2, \cdots, s-1$ there exists an $a_{i}$-chain for $\left(\nu_{i-1}, \nu_{i}\right)$. An $L S$-path is a piecewise linear path. Let $\bar{\Pi}$ (respectively, $\Pi$ ) be the set of all piecewise linear paths $\pi:[0,1]_{\mathbf{Q}} \rightarrow \bar{P} \otimes_{\mathbf{Z}} \mathbf{Q}$ ( respectively, $P \otimes_{\mathbf{Z}} \mathbf{Q}$ ). Unlike the references, e.g. [5,6,7], we consider the same path with different parameterizations as different paths. It is obvious that $\Pi \subseteq \bar{\Pi}$. Let $\mathbf{Z} \bar{\Pi}$ (respectively, $\mathbf{Z} \Pi$ ) be the free $\mathbf{Z}$-module with basis $\bar{\Pi}$ (respectively, $\Pi$ ). Then $\mathbf{Z} \Pi$ is a submodule of $\mathbf{Z} \bar{\Pi}$. For each $i \in I^{\prime}$ and any $\pi \in \bar{\Pi}$ or $\Pi$, define a function

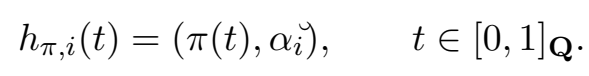


Let $x \in[0,1]_{\mathbf{Q}}$ be any rational number. We define a linear operator $\tau_{i, x}$ on $\mathbf{Z} \bar{\Pi}$ or $\mathbf{Z} \Pi$ as follows.

$$
\tau_{i, x}(\pi)(t)= \begin{cases}\pi(t), & \text { for } 0 \leq t \leq x \\ h_{\pi, i}(x) \alpha_{i}+s_{i}(\pi), & \text { for } x \leq t \leq 1\end{cases}
$$

where $\pi \in \mathbf{Z} \bar{\Pi}$ or $\mathbf{Z} \Pi$. We call $\tau_{i, x}$ an absolute tail-flip operator determined by the simple root $\alpha_{i}$ at $x$, simply tail-flip operator. The following proposition is easily obtained from the definition.

Proposition 3.1. (1) $\tau_{i, x} \tau_{i, y}=\tau_{i, y} \tau_{i, x}$, for any $x, y \in[0,1]_{\mathbf{Q}}$;

(2) $\tau_{i, x} \tau_{i, x}=i d$;

(3) $\tau_{i, x}(\pi)(1)=\pi(1)+\left(h_{\pi, i}(x)-h_{\pi, i}(1)\right) \alpha_{i}$.

Notice that all paths in this paper are piecewise linear paths defined on $[0,1]_{\mathbf{Q}}$.

3.2 Let $m=\min \left\{h_{\pi, i}(t) \mid t \in[0,1]_{\mathbf{Q}}\right\}$ be the absolute minimal value of the function $h_{\pi, i}(t)$. Suppose $L$ is the integral part of $h_{\pi, i}(0)-m$ and $M$ the integral part of $h_{\pi, i}(1)-m$. Then one can define the following operators after Littelmann $([5],[6])$.

Definition 3.1. (1) If $L \leq 0$, then set $E_{i}(\pi)=0$, otherwise $E_{i}(\pi)=\tau_{i, t_{1}} \tau_{i, t_{0}}(\pi)$, where $t_{0}$ is minimal such that $h_{\pi, i}\left(t_{0}\right)=m$ and $t_{1}<t_{0}$ is maximal with $h_{\pi, i}\left(t_{1}\right)=$ $m+1$.

(2) If $M \leq 0$, then let $F_{i}(\pi)=0$, otherwise $F_{i}(\pi)=\tau_{i, t_{1}} \tau_{i, t_{0}}(\pi)$, where $t_{0}$ is maximal such that $h_{\pi, i}\left(t_{0}\right)=m$ and $t_{1}>t_{0}$ is minimal with $h_{\pi, i}\left(t_{1}\right)=m+1$.

If the piecewise linear path $\pi$ satisfies $\pi(0)=0$ and $m$ is an integral number, then $E_{i}(\pi)=e_{\alpha_{i}}(\pi)$ and $F_{i}(\pi)=f_{\alpha_{i}}(\pi)$, where $e_{\alpha_{i}}, f_{\alpha_{i}}$ are the root operators defined in [5].

3.3 After Littelmann, we call $\pi(1)$ the weight of the path $\pi$. The weight of the path $\pi$ is denoted by $v(\pi)$. The following lemma is similar to [5,Lemma 1.4] and [6,Lemma 2.1], so we omit its proof.

Lemma 3.2. (1) If $E_{i}(\pi) \neq 0$, then $v\left(E_{i}(\pi)\right)=v(\pi)+\alpha_{i}$ and if $F_{i}(\pi) \neq 0$, then $v\left(F_{i}(\pi)\right)=v(\pi)-\alpha_{i}$.

(2) If $E_{i}(\pi) \neq 0$, then $E_{i}(\pi)(0)=\pi(0)$ and if $F_{i}(\pi) \neq 0$, then $F_{i}(\pi)(0)=\pi(0)$. 
(3) Let $\rho \in \bar{P}$ be such that $\left(\rho, \alpha_{i}\right)=1$ for $i \in I^{\prime}$, then $E_{i}(\pi)=0$ for all $i \in I^{\prime}$ if and only if the path $\pi+\rho-\pi(0)$ is completely contained in the interior of the dominant Weyl chamber, $F_{i}(\pi)=0$ for all $i \in I^{\prime}$ if and only if the path $\pi+\rho-\pi(1)$ is completely contained in the interior of the dominant Weyl chamber.

(4) If $\pi^{\prime} \neq 0$ is a second path, then $E_{i}(\pi)=\pi^{\prime}$ if and only if $F_{i}\left(\pi^{\prime}\right)=\pi$.

3.4 Let $L, M$ be defined as at the beginning of Section 3.2. Suppose both $L$ and $M$ are larger than zero. For any integer $r$ between 0 and $L$ (respectively, $M)$, set $m_{r}=\min \left\{h_{E_{i}^{r}(\pi), i}(t) \mid t \in[0,1]_{\mathbf{Q}}\right\}$ (respectively, $m_{r}=\min \left\{h_{F_{i}^{r}(\pi), i}(t) \mid t \in\right.$ $\left.[0,1]_{\mathbf{Q}}\right\}$ ). Let $t_{r} \in[0,1]_{\mathbf{Q}}$ be the minimal (resp. maximal) rational numbers satisfying $h_{E_{i}^{r}(\pi), i}\left(t_{r}\right)=m_{r}$. Then the following proposition holds (we make the convention that $\left.F_{i}^{0}=E_{i}^{0}=i d\right)$ :

Proposition 3.2. For any $i \in I^{\prime}$ and $\pi \in \bar{\Pi}$ or $\Pi$, the following statements hold:

(1) The integral part of $h_{E_{i}^{r}(\pi), i}(0)-m_{r}$ is equal to $L-r$ for $r=0, \cdots, L$.

(2) The integral part of $h_{F_{i}^{r}(\pi), i}(1)-m_{r}$ is equal to $M-r$ for $r=0, \cdots, M$.

(3) $E_{i}^{r}(\pi)=\tau_{i, t_{r}} \tau_{i, t_{0}}(\pi)$, for $r=0, \cdots, L$.

(4) $F_{i}^{r}(\pi)=\tau_{i, t_{r}} \tau_{i, t_{0}}(\pi)$, for $r=0, \cdots, M$.

Proof. We only give the proof of (1) and (4). One can prove (2) and (3) similarly.

(1) We shall use induction on $r$. Suppose that statement is true on $r$, i.e., the integral part $h_{E_{i}^{r}(\pi), i}(0)-m_{r}$ is equal to $L-r$. Since $E_{i}^{r+1}(\pi)=E_{i}\left(E_{i}^{r}(\pi)\right)$, $E_{i}^{r+1}(\pi)(0)$ is equal to $E_{i}^{r}(\pi)(0)$ by Lemma 3.2. Hence $h_{E_{i}^{r+1}(\pi), i}(0)=h_{E_{i}^{r}(\pi), i}(0)$. It is obvious that $m_{r+1}=m_{r}+1$. So $h_{E_{i}^{r+1}(\pi), i}(0)-m_{r+1}=h_{E_{i}^{r}(\pi), i}(0)-m_{r}-1$. Consequently the integral part of $h_{E_{i}^{r+1}(\pi), i}(0)-m_{r+1}$ is equal to $L-r-1$.

(4) Again we shall use induction on $r$. Assume that the statement holds for $r \in \mathbf{N}$. Then $F_{i}^{r+1}(\pi)=F_{i}\left(F_{i}^{r}(\pi)\right)=F_{i}\left(\tau_{i, t_{r}} \tau_{i, t_{0}}(\pi)\right)=\tau_{i, t_{r+1}} \tau_{i, t_{r}}\left(\tau_{i, t_{r}} \tau_{i, t_{0}}(\pi)\right)=$ $\tau_{i, t_{r+1}} \tau_{i, t_{0}}(\pi)$ via the induction hypothesis and Proposition 3.1.

3.5 Let $\Pi_{\text {int }}$ be the set of all piecewise paths satisfying $\pi(0)=0$ and $\pi(1) \in \bar{P}$. Let $\mathbf{Z} \Pi_{\text {int }}$ be a $\mathbf{Z}$ module with the basis $\Pi_{\text {int }}$. For any $\mathbf{Z}$ module $M$, the set of endomorphisms of $M$ is denoted by EndM.

Suppose $\pi$ is a piecewise path such that $\pi(0)=0, n:=\left(\pi(1), \alpha_{i}^{y}\right)$ is an integer. If $n \geq 0$, then there exists $y \in[0,1]_{\mathbf{Q}}$ maximal with $h_{\pi, i}(y)=m$, the absolute 
minimum of $h_{\pi, i}$. Let $q>y$ be maximal such that $h_{\pi, i}(q)=m+n$. If $n<0$, then there exist $x, p \in[0,1]_{\mathbf{Q}}$ such that $x$ is minimal with $h_{\pi, i}(x)=m$ and $p<x$ maximal with $h_{\pi, i}(p)=m-n$. Define $\tilde{S}_{i}(\pi)$ as follows:

$$
\tilde{S}_{i}(\pi)=\left\{\begin{array}{l}
\tau_{i, y} \tau_{i, q}(\pi), \text { for } n \geq 0 \\
\tau_{i, x} \tau_{i, p}(\pi), \text { for } n<0
\end{array}\right.
$$

Then $\tilde{S}_{i}{ }^{2}=i d$. Using the above propositions and [6, Theorem 8.1], one can easily prove that the map $\psi$, which is defined via $\psi\left(s_{i}\right)=\tilde{S}_{i}$ on the simple reflections in Weyl group $\bar{W}$, can be extended to a representation $\bar{W} \rightarrow E n d \mathbf{Z} \Pi_{i n t}$ such that $w(\pi)(1)=w(\pi(1))$ for $\pi \in \Pi_{\text {int }}$ and $w \in \bar{W}$.

Proposition 3.3. Let $\pi$ be an $L S$ path such that $v(\pi)$ is a weight of some $\mathcal{G}^{\prime}$ module $V$. Assume there is a rational number $u$ such that $h_{\pi, i}(u)$ is an integer number, and $h_{\pi, i}(u) \leq h_{\pi, i}(t)$ for all $t \geq u$. Then $v\left(\tau_{i, u}(\pi)\right)$ is a weight of $V$.

Proof. Let $n:=\left(\pi(1), \alpha_{i}^{y}\right)$ be the integral number. From the above discussion, we know that $s_{i}(\pi)(1)=\pi(1)-n \alpha_{i}$ is a weight of $V$. If $n>0$, then $h_{\pi, i}(y)=$ $m \leq h_{\pi, i}(u) \leq h_{\pi, i}(t) \leq n$ implies $0 \leq n-h_{\pi, i}(u) \leq n-m$. By [6,Lemma 2.1], $v\left(f_{\alpha_{i}}^{n-m}(\pi)\right)=\pi(1)-(n-m) \alpha_{i}$ is a weight of $V$. Since $v\left(\tau_{i, u}(\pi)\right)=\pi(1)-(n-$ $\left.h_{\pi, i}(u)\right) \alpha_{i}$, it is a weight of $V$ by [3,Proposition 3.6]. If $n \leq 0$, then $m=h_{\pi, i}(x) \leq$ $h_{\pi, i}(u) \leq n$ by the assumption. From this we get $m-n \leq h_{\pi, i}(u)-n \leq 0$. Then $v\left(e_{\alpha_{i}}^{n-m}(\pi)\right)=\pi(1)-(m-n) \alpha_{i}$ is a weight of $V$ by [6, Lemma 2.1]. Hence $v\left(\tau_{i, u}(\pi)\right)=\pi(1)-\left(n-h_{\pi, i}(u)\right) \alpha_{i}$ is a weight of $V$.

3.6 For any path $\pi \in \bar{\Pi}$ (or $\Pi$ ), denote by $\pi^{*}(t)=-\pi(1-t)$ the dual path of $\pi$. The vector space $\mathbf{Z} \Pi$ and $\mathbf{Z} \bar{\Pi}$ are algebras under the product $\otimes$ defined as

$$
\pi_{1} \otimes \pi_{2}(t):= \begin{cases}\pi_{1}(2 t), & \text { for } 0 \leq t \leq \frac{1}{2} \\ \pi_{1}(1)+\pi(2 t-1), & \text { for } \frac{1}{2} \leq t \leq 1\end{cases}
$$

It is obvious that $\mathbf{Z} \Pi$ is a subalgebra of $\mathbf{Z} \bar{\Pi}$ and $*$ is an involution of the algebra $\mathbf{Z} \bar{\Pi}$ (or $\mathbf{Z} \Pi$ ).

Let $\mathcal{A}_{t}$ be the algebra generated by all tail-flip operators $\tau_{i, x}$ over $\mathbf{Z}$. Then $\mathbf{Z} \bar{\Pi}$ and $\mathbf{Z} \Pi$ become $\mathcal{A}_{t}$ modules respectively. View $\bar{P}_{\mathbf{Q}}:=\bar{P} \otimes_{\mathbf{Z}} \mathbf{Q}$ or $P_{\mathbf{Q}}:=P \otimes_{\mathbf{Z}} \mathbf{Q}$ as constant paths. Then $\bar{P}_{\mathbf{Q}}$ (respect. $P_{\mathbf{Q}}$ ) becomes an $\mathcal{A}_{t}$ submodule of $\mathbf{Z} \bar{\Pi}$ (respectively, Z⿱ $\Pi$ ). This submodule is stable under the operators $E_{i}^{\prime} s$ and $F_{i}^{\prime} s$. Let us use $\mathcal{A}_{e}$ (respectively, $\mathcal{A}_{f}$ ) to denote the algebra generated by $E_{i}^{\prime} s$ (respectively, $F_{i}^{\prime} s$ ). Then $\mathbf{Z} \bar{\Pi} / \bar{P}_{\mathbf{Q}}$ and $\mathbf{Z} \Pi / P_{\mathbf{Q}}$ are modules over $\mathcal{A}_{e}$ and $\mathcal{A}_{f}$. Suppose $\mathcal{A}$ 
is the algebra generated by $\mathcal{A}_{e} \cup \mathcal{A}_{f}$. Then $\mathbf{Z} \bar{\Pi} / \bar{P}_{\mathbf{Q}}$ and $\mathbf{Z} \Pi / P_{\mathbf{Q}}$ are also modules over $\mathcal{A}$, which can be identified with the path model in [5]. Obviously, $\mathbf{Z} \bar{\Pi} / \bar{P}_{\mathbf{Q}}$

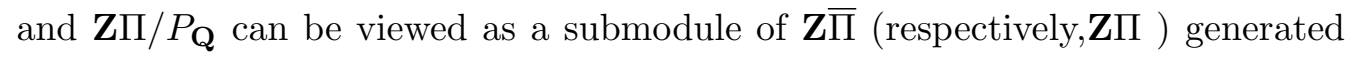
by all piecewise linear paths starting from 0 . To simplify the notation, we use $\overline{\mathcal{P}}$ (respectively, $\mathcal{P}$ ) to denote the factor module $\mathbf{Z} \bar{\Pi} / \bar{P}_{\mathbf{Q}}$ ( respectively, $\mathbf{Z} \Pi / P_{\mathbf{Q}}$ ). The image of a path $\pi$ in $\overline{\mathcal{P}}$ (respectively, $\mathcal{P}$ ) is still denoted by $\pi$.

Proposition 3.4. For any $\pi \in \overline{\mathcal{P}}$ or $\mathcal{P}$, and any $i \in I^{\prime}$, the following statements hold.
(1) $\tau_{i, x}\left(\pi^{*}\right)=\left(\tau_{i, 1-x}(\pi)\right)^{*}$.
(2) $\tau_{i, 1-x}\left(\tau_{i, x}(\pi)^{*}\right)=s_{i}(\pi)$.
(3) $E_{i}\left(\pi^{*}\right)=F_{i}(\pi)^{*}$ and $F_{i}\left(\pi^{*}\right)=E_{i}(\pi)^{*}$.

3.7 Following Greenstein and Lampron $([2])$, we use $\xi$ to denote the canonical projection from $\bar{P}$ to $\bar{P} / \mathbf{Z} \delta$. Define $(\Xi \pi)(t):=\xi(\pi(t))$ for any $\pi \in \overline{\mathcal{P}}$ and any $t \in[0,1]_{\mathbf{Q}}$. By the following proposition, $\Xi$ is an $\mathcal{A}_{t}\left(\mathcal{A}_{e}, \mathcal{A}_{f}, \mathcal{A}\right)$ module homomorphism from $\overline{\mathcal{P}}$ to $\mathcal{P}$.

Proposition 3.5. For any $\pi \in \overline{\mathcal{P}}$, and any $i \in I^{\prime}, \Xi\left(\tau_{i, x}(\pi)\right)=\tau_{i, x}(\Xi(\pi))$. Consequently, $\Xi\left(E_{i}(\pi)\right)=E_{i}(\Xi(\pi))$ and $\Xi\left(F_{i}(\pi)\right)=F_{i}(\Xi(\pi))$.

Proof. The proof of $\Xi\left(E_{i}(\pi)\right)=E_{i}(\Xi(\pi))$ and $\Xi\left(F_{i}(\pi)\right)=F_{i}(\Xi(\pi))$ has been given in [2]. We only need to prove that $\Xi\left(\tau_{i, x}(\pi)\right)=\tau_{i, x}(\Xi(\pi))$. The proof is similar to the proof of [2, Lemma 5.6].

\section{A first CHARACTER FORMUla}

For any piecewise linear path $\pi \in \overline{\mathcal{P}}$, the submodule $\mathcal{A}_{t} \pi$, which is generated by the path $\pi$ over $\mathcal{A}_{t}$, unlike $\mathcal{A} \pi$, contains too many paths for our purpose to prove the Weyl character formula. So we need to define new "tail-flip" operators to cut down the number of paths. Let $h_{\pi, i}(t)$ be the function defined in Section 3 .

Definition 4.1. Let $\pi$ be a piecewise linear path and $x \in[0,1]_{\mathbf{Q}}$. Then we define $T_{i, x}(\pi)$ as follows:

In the case $h_{\pi, i}(x)$ is not an integer, then $T_{i, x}(\pi)=0$ : 
In the case $h_{\pi, i}(x)>h_{\pi, i}(t)$ for some $t>x$ and $x \neq 0$, then $T_{i, x}(\pi)=0$;

In the case $x=0, T_{i, 0}(\pi)=s_{i}(\pi)$;

In the case $h_{\pi, i}(x)$ is an integer, $h_{\pi, i}(x) \leq h_{\pi, i}(t)$ for $t>x$, then

$$
T_{i, x}(\pi)(t)= \begin{cases}\pi(t), & \text { for } 0 \leq t \leq x \\ h_{\pi, i}(x) \alpha_{i}+s_{i}(\pi), & \text { for } x \leq t \leq 1\end{cases}
$$

Let $\mathcal{B}$ be the algebra generated by $\left\{T_{i, x} \mid i \in I^{\prime}, x \in[0,1]_{\mathbf{Q}}\right\}$ over $\mathbf{Z}$, and $\mathcal{B}_{m}$ be the monoid generated by $\left\{T_{i, x} \mid i \in I^{\prime}, x \in[0,1]_{\mathbf{Q}}\right\}$. Since $\mathcal{B}$ is a subalgebra of $\mathcal{A}_{t}, \overline{\mathcal{P}}$ and $\mathcal{P}$ become $\mathcal{B}$ modules. For any piecewise linear path $\pi$, the module generated by $\pi$ is denoted by $\mathcal{B} \pi$, and the set of all paths contained in $\mathcal{B} \pi$ is denoted by $\mathcal{B}(\pi)$. It is obvious that $\mathcal{B}(\pi)=\left\{b \pi \mid b \in \mathcal{B}_{m}\right\}$.

Example Let $\mathcal{T}$ be the group generated by $\left\{T_{i, 0} \mid i \in I^{\prime}\right\}$. Then $\mathcal{T} \pi=\bar{W} \pi$ for any path $\pi$, where $(w(\pi))(t):=w(\pi(t))$ for $t \in[0,1]_{\mathbf{Q}}$. So $\sum_{\eta \in B} e^{\eta(1)}$ is stable under the action of the Weyl group $\bar{W}$, whenever the set of paths $B$ is stable under the action of $\mathcal{B}_{m}$.

Proposition 4.1. Let $\pi_{\lambda}(t)=t \lambda, t \in[0,1]_{\mathbf{Q}}$, where $\lambda$ is a dominant weight. If $B$ is the set of all paths contained in $\mathcal{B} \pi_{\lambda}$, then Char $B$ is the character formula of a simple module with highest weight $\lambda$.

Proof. Suppose $\pi$ is a piecewise linear path such that $\pi(0)=0$ and $\pi(1)$ is an integral weight. Then there exists $y \in[0,1]_{\mathbf{Q}}$ maximal with $h_{\pi, i}(y)=m$, the absolute minimum of $h_{\pi, i}$. Let $q>y$ be maximal such that $h_{\pi, i}(q)=m+1$. Similarly, there exist $x, p \in[0,1]_{\mathbf{Q}}$ such that $x$ is minimal with $h_{\pi, i}(x)=m$ and $p<x$ maximal with $h_{\pi, i}(p)=m+1$. From the definition, we get $e_{\alpha_{i}}(\pi)=$ $T_{0, i} T_{p, i} T_{0, i} T_{x, i}(\pi)$ and $f_{\alpha_{i}}(\pi)=T_{0, i} T_{q, i} T_{0, i} T_{y, i}(\pi)$. Thus every set of pahts, which is stable under the action of all tail-flip operators, is also stable under the action of all root operators defined in $[5,6,7]$.

On the other hand, suppose $V_{\lambda}$ is a simple $\mathcal{G}^{\prime}$-module determined by a dominant weight $\lambda$. Then $\eta(1)$ is a weight of $V_{\lambda}$ for any piecewise linear path $\eta$ in $\mathcal{B} \pi_{\lambda}$ by Proposition 3.3. Thus $\operatorname{Char}_{\lambda}=\sum_{\eta \in B} e^{\eta(1)}$.

Proposition 4.2. Let $\pi$ be a piecewise linear path satisfying $(\pi(t) \mid \delta)=1$ for all $t \in[0,1]_{\mathbf{Q}}$. Then the rank of $\mathcal{B} \pi$ is finite. 
Proof. To prove this proposition, we use the theory of galleries ([1]). Notice that $\bar{W}$ can be viewed as a group generated by the affine reflections $s_{i, k}$, where $s_{i, k}(\lambda)=$ $s_{i}(\lambda)+k \alpha_{i}$ for all $k \in \mathbf{Z}$ and $i \in I^{\prime}, \lambda \in \mathcal{H}^{\prime *}([3])$. The hyperplanes $H_{\alpha, k}:=$ $\left\{\lambda \in \mathcal{H}^{\prime *} \mid\left(\lambda, \alpha^{4}\right)=k\right\}$ for $\alpha \in \Phi^{+}$subdivide the real vector space $\mathcal{H}_{\mathcal{R}}^{\prime *}$ into open regions, called alcoves. Each alcove $A$ is given by $A=\left\{\lambda \in \mathcal{H}_{\mathcal{R}}^{* *} \mid m_{\alpha}<(\lambda, \alpha)<\right.$ $m_{\alpha}+1$, for all $\left.\alpha \in \Phi^{+}\right\}$, where $m_{\alpha}$ are some integal numbers. Following Gaussent and Littelmann, a gallery of alcoves of length $r$ is a sequence $\left(\Delta_{0}, \cdots, \Delta_{r}\right)$ of alcoves such that $\Delta_{i}$ and $\Delta_{i+1}$ are adjacent for $1 \leq i \leq r$. Every piecewise path can be contained in one of such gallery. Suppose $\pi$ is contained in a gallery $\left(\Delta_{0}, \cdots, \Delta_{r}\right)$. If the alcoves $\Delta_{t}$ and $\Delta_{t+1}$ have a common face on the hyperplane $H_{i, m}=\left\{\lambda \mid\left(\lambda, \alpha^{y}\right)=m\right\}$, where $m=h_{\pi, i}(x)$, then $T_{x, i}(\pi)$ is contained in the gallery $\left(\Delta_{0}^{\prime}, \cdots, \Delta_{r}^{\prime}\right)$, where $\Delta_{i}^{\prime}=\Delta_{i}$ for $i=0,1, \cdots, t$, and $\Delta_{j}^{\prime}=s_{i, m}\left(\Delta_{j}\right)$ for $j=t+1, \cdots, r$. For any piecewise path $\pi$ satisfying $(\pi(t) \mid \delta)=1$, we can choose these galleries $\left(\Delta_{0}, \cdots, \Delta_{r}\right)$ containing the path $\pi$ with $r$ minimal. Then the number of such galleries is finite. Thus, the number of galleries containing all paths $T_{x_{1}, i_{1}} \cdots T_{x_{j}, i_{j}}(\pi)$ with minimal length are finite too. So the rank of $\mathcal{B} \pi$ is finite.

Recall that the product of two paths $\pi_{1}, \pi_{2}$ is defined as follows:

$$
\pi_{1} \otimes \pi_{2}(t):=\left\{\begin{array}{ll}
\pi_{1}(2 t), & \text { for } 0 \leq t \leq \frac{1}{2} \\
\pi_{1}(1)+\pi(2 t-1), & \text { for } \frac{1}{2} \leq t \leq 1
\end{array} .\right.
$$

We define $\pi_{1} \otimes \cdots \otimes \pi_{k}:=\left(\pi_{1} \otimes \cdots \otimes \pi_{k-1}\right) \otimes \pi_{k}$ inductively if $k>2$. If $X_{i}(i=1,2, \cdots, k)$ are sets of paths, then $X_{1} \otimes \cdots \otimes X_{k}:=\left\{\pi_{1} \otimes \cdots \otimes \pi_{k} \mid \pi_{i} \in X_{i}\right\}$. We use $\mathcal{A}_{t}(\pi)$ to denote the set of all paths contained in the module $\mathcal{A}_{t} \pi$.

Proposition 4.3. Let $\pi_{1} \cdots \pi_{r} \in \overline{\mathcal{P}}$, or $\mathcal{P}$ such that $\pi=\pi_{1} \otimes \cdots \otimes \pi_{r}$. Then

$$
\begin{aligned}
& \text { (1) } \mathcal{A}_{t}(\pi)=\mathcal{A}_{t}\left(\pi_{1}\right) \otimes \cdots \otimes \mathcal{A}_{t}\left(\pi_{r}\right) . \\
& \text { (2) } \mathcal{B}(\pi) \subseteq \mathcal{B}\left(\pi_{1}\right) \otimes \cdots \otimes \mathcal{B}\left(\pi_{r}\right) .
\end{aligned}
$$

Proof. (1) To simplify the notation, we give only the proof for the case $r=2$, the proof for $r>2$ is similar. For any $0 \leq x<\frac{1}{2}, \tau_{i, x}(\pi)=\tau_{i, x}\left(\pi_{1}\right) \otimes \tau_{i, \frac{1}{2}}\left(\pi_{2}\right)$. If $x \geq \frac{1}{2}$, then $\tau_{i, x}(\pi)=\pi_{1} \otimes \tau_{i, 2 x-1}\left(\pi_{2}\right)$. So $\mathcal{A}_{t}(\pi) \subseteq \mathcal{A}_{t}\left(\pi_{1}\right) \otimes \mathcal{A}_{t}\left(\pi_{2}\right)$. On the other hand, we have

$$
\begin{aligned}
& \tau_{i_{1}, x_{1}} \cdots \tau_{i_{k}, x_{k}}\left(\pi_{1}\right) \otimes \tau_{j_{1}, y_{1}} \cdots \tau_{j_{s}, y_{s}}\left(\pi_{2}\right) \\
& =\tau_{i_{1}, \frac{1}{2} x_{1}} \cdots \tau_{i_{k}, \frac{1}{2} x_{k}} \tau_{j_{1}, \frac{1}{2}\left(2 y_{1}+1\right)} \cdots \tau_{j_{s}, \frac{1}{2}\left(2 y_{s}+1\right)}(\pi)
\end{aligned}
$$


for any $\tau_{i_{1}, x_{1}} \cdots \tau_{i_{k}, x_{k}}\left(\pi_{1}\right) \in \mathcal{A}_{t} \pi_{1}$, and $\tau_{j_{1}, y_{1}} \cdots \tau_{j_{s}, y_{s}}\left(\pi_{2}\right) \in \mathcal{A}_{t} \pi_{2}$. Hence $\mathcal{A}_{t}(\pi) \supseteq$ $\mathcal{A}_{t}\left(\pi_{1}\right) \otimes \mathcal{A}_{t}\left(\pi_{2}\right)$ and (1) holds.

(2) Similarly to (1), we only give the proof for the case $r=2$. If $T_{i, x}\left(\pi_{1} \otimes \pi_{2}\right)=$ 0 , then $T_{i, x}\left(\pi_{1} \otimes \pi_{2}\right) \in \mathcal{B}\left(\pi_{1}\right) \otimes \mathcal{B}\left(\pi_{2}\right)$. In the following, we assume that $T_{i, x}\left(\pi_{1} \otimes\right.$ $\left.\pi_{2}\right) \neq 0$. In the case $x=0, T_{i, 0}\left(\pi_{1} \otimes \pi_{2}\right)=T_{i, 0}\left(\pi_{1}\right) \otimes T_{i, 0}\left(\pi_{2}\right) \in \mathcal{B}\left(\pi_{1}\right) \otimes \mathcal{B}\left(\pi_{2}\right) ;$ In the case $0<x \leq \frac{1}{2}$, since $h_{\pi_{1}, i}(x) \leq h_{\pi_{1}, i}(t)$ for $t>x, T_{i, x}\left(\pi_{1} \otimes \pi_{2}\right)=T_{i, 2 x}\left(\pi_{1}\right) \otimes$ $T_{i, 0}\left(\pi_{2}\right) \in \mathcal{B}\left(\pi_{1}\right) \otimes \mathcal{B}\left(\pi_{2}\right)$. In the case $\frac{1}{2}<x \leq 1$, if $h_{\pi_{2}, i}(2 x-1)>h_{\pi_{2}, i}(t)$ for some $t>2 x-1$, then $h_{\pi_{1}, i}(1)+h_{\pi_{2}, i}(2 x-1)>h_{\pi_{1}, i}(1)+h_{\pi_{2}, i}(t)$ and $T_{i, x}\left(\pi_{1} \otimes \pi_{2}\right)=0$, which is contradict to our assumption. Hence $h_{\pi_{2}, i}(2 x-1) \leq h_{\pi_{2}, i}(t)$ for all $t \geq 2 x-1$. Thus $T_{i, x}\left(\pi_{1} \otimes \pi_{2}\right)=\pi_{1} \otimes T_{i, 2 x-1}\left(\pi_{2}\right) \in \mathcal{B}\left(\pi_{1}\right) \otimes \mathcal{B}\left(\pi_{2}\right)$. Consequently (2) holds.

If $B \subseteq \overline{\mathcal{P}}$ is a subset, which is stable under the action of all operators $T_{i, x}$, then we have already seen that its character $\operatorname{Char} B:=\sum_{\eta \in B} e^{\eta(1)}$ is stable under the action of the Weyl group $\bar{W}$. In fact, $C h a r B$ can be computed by the following path version of Weyl's character formula.

Theorem 4.2. (1) Let $\Pi_{0}^{+}$be the set of the piecewise linear paths such that Im is in the interior of $\mathcal{C}$ (for $t>0$ ), where $\mathcal{C}$ is the Tits cone of a nontwised affine Kac-Moody algebra $\mathcal{G}^{\prime}$. Suppose $B$ is a set of piecewise linear paths, which is stable under the action of operators $T_{i, x}$, where $x \in[0,1]_{\mathbf{Q}}$ and $i \in I^{\prime}$. Then

$$
\left(\sum_{w \in \bar{W}} \operatorname{sgn}(w) e^{w(\rho)}\right) C h a r B=\sum_{\eta \in B, \rho \otimes \eta \in \Pi_{0}^{+}}\left(\sum_{w \in \bar{W}} \operatorname{sgn}(w) e^{w(\rho+\eta(1))}\right) .
$$

(2) For any dominant weight $\mu$, let $V_{\mu}$ be the corresponding irreducible $\mathcal{G}^{\prime}$ representation, then

$$
\operatorname{Char} B=\sum_{\eta \in B, \rho \otimes \eta \in \Pi_{0}^{+}} \operatorname{Char}_{\eta(1)}
$$

Proof. (2) of this proposition follows from (1). To prove (1), we only need to compare the coefficients of the terms corresponding to dominant weights, i. e. we have to prove for $\Omega:=\{(w, \pi) \mid w \in \bar{W}, \pi \in B, w(\rho)+\pi(1) \in \bar{P}\}$, 


$$
\sum_{(w, \pi) \in \Omega} \operatorname{sgn}(w) e^{w(\rho)+\pi(1)}=\sum_{\eta \in B, \rho \otimes \eta \in \Pi_{0}^{+}} e^{\rho+\eta(1))} .
$$

Let $\Omega_{0}:=\left\{(i d, \pi) \in \Omega \mid \rho \otimes \pi \in \Pi_{0}^{+}\right\}$. Set $\Omega^{\prime}=\Omega-\Omega_{0}$. To prove the proposition, we have to show that

$$
\sum_{(w, \pi) \in \Omega^{\prime}} \operatorname{sgn}(w) e^{w(\rho)+\pi(1)}=0 .
$$

We will define an involution $\phi: \Omega^{\prime} \rightarrow \Omega^{\prime}$ such that $\phi(w, \pi)=\left(w^{\prime}, \pi^{\prime}\right)$ has the property: $\operatorname{sgn}(w)=-\operatorname{sgn}\left(w^{\prime}\right)$ and $w(\rho)+\pi(1)=w^{\prime}(\rho)+\pi^{\prime}(1)$. If such an involution exists, then it is obvious that

$$
\sum_{(w, \pi) \in \Omega^{\prime}} \operatorname{sgn}(w) e^{w(\rho)+\pi(1)}=0 .
$$

The construction of the involution. Suppose $(w, \pi) \in \Omega^{\prime}$ is such that $w$ is not the identity. Since $w(\rho)+\pi \in P^{+}$, the path $w(\rho) \otimes \pi$ has to meet at least once a proper face of the dominant Weyl's chamber $C$. If $w$ is the identity, then $w(\rho) \otimes \pi$ also has to meet a proper face $F$ of $C$, the pair would otherwise be an element of $\Omega_{0}$.

For a proper face $F$ of $C$ denote by $\Omega^{\prime}(F)$ the set of pairs $(w, \pi) \in \Omega^{\prime}$ which meet $F$ as the last face. More precisely: $w(\rho) \otimes \pi$ meets $F$, and if $t_{0} \in[0,1]_{\mathbf{Q}}$ is maximal with property such that $w(\rho)+\pi\left(t_{0}\right) \in F$, then $w(\rho)+\pi\left(t_{0}\right)$ is in the interior of $F$, and $w(\rho)+\pi(t)$ is in the interior of $C$ for all $t>t_{0}$.

The set $\Omega^{\prime}$ is obviously the disjoint union of the $\Omega^{\prime}(F)$, so it is sufficient to define an involution for such an $\Omega^{\prime}(F)$. Let $\alpha_{i}$ be a simple root orthogonal to $F$. For $(w, \pi) \in \Omega^{\prime}(F)$ set $n:=\left(w(\rho), \alpha_{i}^{y}\right)$, note that $n \neq 0$.

Without loss generality, we can assume $n>0$. Then the function $h_{\pi, i}\left(t_{0}\right)=$ $-n$. It is easy to prove $T_{i, 0} T_{i, t_{0}}(\pi)(1)=\pi(1)+n \alpha_{i}$. It follows that $w(\rho)+\pi(1)=$ $s_{i} w(\rho)+T_{i, 0} T_{i, t_{0}}(\pi)(1)$. Further, $w(\rho) \otimes \pi(t)=s_{i} w(\rho) \otimes T_{i, 0} T_{i, t_{0}}(\pi)(t)$ for all $t>t_{0}$. Hence $\phi(w, \pi)=\left(s_{i} w, T_{i, t_{0}}(\pi)\right) \in \Omega^{\prime}(F)$.

In the following proposition, we use $\mathcal{P}^{+}$to denote the set of all piecewise linear paths satisfying $\left(\pi(t), \alpha^{y} \geq 0\right.$ for any positive root $\alpha$.

Proposition 4.4. Suppose $\pi_{1}, \pi_{2} \in \mathcal{P}^{+}$. Then

$$
\mathcal{B}\left(\pi_{1}\right) \otimes \mathcal{B}\left(\pi_{2}\right)=\cup \mathcal{B}\left(\pi_{1} \otimes \eta\right),
$$


where the union runs over all paths $\eta \in \mathcal{B} \pi_{2}$ such that $\pi_{1} \otimes \eta \in \mathcal{P}^{+}$.

Proof. Let $X=\cup \mathcal{B}\left(\pi_{1} \otimes \eta\right)$, the right side of equation (4.1). Since $T_{i, x} \pi_{1} \otimes \pi_{2}=$ $T_{i, 0} T_{i, \frac{1}{2}} T_{i, 0} T_{i, \frac{x}{2}}\left(\pi_{1} \otimes \pi_{2}\right), T_{i, x} \pi_{1} \otimes \pi_{2} \in X$ for $i \in I^{\prime}$ and $x \in[0,1]_{\mathbf{Q}}$. Now assume that $T_{i_{1}, x_{1}} \cdots T_{i_{k}, x_{k}} \pi_{1} \otimes \pi_{2} \in X$ for any $i_{1}, \cdots, i_{k} \in I^{\prime}$ and any $x_{1}, \cdots, x_{k} \in$ $[0,1]_{\mathbf{Q}}$, where $k \geq 1$. Let $b=T_{i_{1}, x_{1}} \cdots T_{i_{k}, x_{k}}, i \in I^{\prime}$ and $x \in[0,1]_{\mathbf{Q}}$. Then $T_{i, x} b \pi_{1} \otimes \pi_{2}=T_{i, 0} T_{i, \frac{1}{2}} T_{i, 0} T_{i, \frac{x}{2}}\left(b \pi_{1} \otimes \pi_{2}\right) \in X$ by the assumption. By now we have already proved that $b \pi_{1} \otimes \pi_{2} \in X$ for any $b \in \mathcal{B}_{m}$. Next, we assume that $b_{1} \pi_{1} \otimes b_{2} \pi_{2} \in X$ for some $b_{1}, b_{2} \in \mathcal{B}_{m}$, where $b_{2}=T_{i_{1}, x_{1}} \cdots T_{i_{k}, x_{k}}$ for some $i_{1}, \cdots, i_{k} \in I^{\prime}$ and some $x_{1}, \cdots, x_{k} \in[0,1]_{\mathbf{Q}}$, and $k \geq 1$. Set $b_{2}^{\prime}=T_{j, y} b_{2}$, where $j \in I^{\prime}$ and $x \in[0,1]_{\mathbf{Q}}$. Consider the path $b_{1} \pi_{1} \otimes b_{2}^{\prime} \pi_{2}$. If $y \neq 0$, then $b_{1} \pi_{1} \otimes b_{2}^{\prime} \pi_{2}=T_{j, \frac{1}{2}(y+1)}\left(b_{1} \pi_{1} \otimes b_{2} \pi_{2}\right) \in X$ by the assumption. If $y=0$, then $b_{1} \pi_{1} \otimes b_{2}^{\prime} \pi_{2}=T_{j, 0}\left(T_{j, 0} b_{1} \pi_{1} \otimes b_{2} \pi_{2}\right) \in X$. So $\mathcal{B}\left(\pi_{1}\right) \otimes \mathcal{B}\left(\pi_{2}\right) \subseteq X$.

On the other hand, let $\pi_{1} \otimes \eta$ be a piecewise linear path for some $\eta \in \mathcal{B}\left(\pi_{2}\right)$, and $\pi_{1} \otimes \eta \in \mathcal{P}^{+}$. Suppose $b\left(\pi_{1} \otimes \eta\right) \in \mathcal{B}\left(\pi_{1}\right) \otimes \mathcal{B}\left(\pi_{2}\right)$ for $b=T_{i_{1}, x_{1}} \cdots T_{i_{k}, x_{k}}$, where $i_{1}, \cdots, i_{k} \in I^{\prime}$ and $x_{1}, \cdots, x_{k} \in[0,1]_{\mathbf{Q}}$. Let $b^{\prime}=T_{j, x} b$, where $j \in I^{\prime}$ and $x \in[0,1]_{\mathbf{Q}}$. Consider the element $b^{\prime}\left(\pi_{1} \otimes \eta\right)$. If $b^{\prime}\left(\pi_{1} \otimes \eta\right) \neq 0$, and $b\left(\pi_{1} \otimes \eta\right)=$ $\eta_{1} \otimes \eta_{2} \in \mathcal{B}\left(\pi_{1}\right) \otimes \mathcal{B}\left(\pi_{2}\right)$, where $\eta_{i} \in \mathcal{B}\left(\pi_{i}\right)$, then

$$
T_{j, x}\left(\eta_{1} \otimes \eta_{2}\right)=\left\{\begin{array}{l}
T_{j, 0} \eta_{1} \otimes T_{j, 0} \eta_{2}, \quad x=0 \\
T_{j, 2 x} \eta_{1} \otimes T_{j, 0} \eta_{2}, 0<x \leq \frac{1}{2} \\
\eta_{1} \otimes T_{j,(2 x-1)} \eta_{2}, \frac{1}{2}<x \leq 1
\end{array}\right.
$$

Hence $b^{\prime}\left(\pi_{1} \otimes \eta\right) \in \mathcal{B}\left(\pi_{1}\right) \otimes \mathcal{B}\left(\pi_{2}\right)$. Consequently, $\mathcal{B}\left(\pi_{1}\right) \otimes \mathcal{B}\left(\pi_{2}\right)=\cup \mathcal{B}\left(\pi_{1} \otimes \eta\right)$.

From Proposition 4.4, we can easily prove the following.

Corollary 4.1. Generalized Littlewood-Richardson Rule. For dominant weights $\lambda$, $\mu$, let $\pi_{1}, \pi_{2} \in \mathcal{P}^{+}$be such that $\pi_{1}(1)=\lambda$ and $\pi_{2}(1)=\mu$. Then the tensor product of irreducible representations $V_{\lambda}$ and $V_{\mu}$ of heightest weight $\lambda, \mu$ is isomorphic to the direct sum

$$
V_{\lambda} \otimes V_{\mu} \simeq \oplus V_{\lambda+\eta(1)}
$$

where the sum runs over all paths $\eta \in \mathcal{B}\left(\pi_{2}\right)$ such that $\pi_{1} \otimes \eta \in \mathcal{P}^{+}$.

Proof. Recall that $\mathcal{B}(\pi)$ is the set of paths contained in $\mathcal{B} \pi$. Then

$$
\operatorname{Char}\left(\mathcal{B}\left(\pi_{1}\right) \otimes \mathcal{B}\left(\pi_{2}\right)\right)=\operatorname{Char} \mathcal{B}\left(\pi_{1}\right) \operatorname{Char} \mathcal{B}\left(\pi_{2}\right)=\operatorname{Char}\left(V_{\pi_{1}(1)} \otimes V_{\pi_{2}(1)}\right) .
$$


Hence this corollary holds.

\section{ACKNOWLEDGMENT}

The author thanks the referees for their useful comments on this paper. He also thanks Professor Robert J. Stanton and Professor Dan Barbasch for their kind help, and the CSC for the support and the Mathematics Department of Wuppertal University for the hospitality during the year 2003/2004.

\section{REFERENCES}

1. S. Gaussent, P. Littelmann, LS-Galleries, the path module and MV-cycles, arXiv:math.RT/0307122 v2, 10 Jul 2003.

2. J. Greenstein, P. Lamprou, Path Model For Quantum Loop Modules Of Fundamental Type, arXiv:math.QA/0307049 v1, 3 Jul 2003.

3. V.G.Kac, Infinte dimensionsal Lie algebras, Cambridge Univ. Press, Cambridge, United Kingdom, 1992.

4. P. Littelmann, A generalization of the Littlewood-Richardson rule, Joural of algebra 130(1990), 328-368.

5. P. Littelmann, A Littlewood-Richardson rule for symmetrizable Kac-Moody algebras, Inventiones Mathematicae 116(1994), 329-346.

6. P. Littelmann, Paths and root operators in representation theorey, Annals of Math 142(1995), 499-525.

7. P. Littelmann, Contracting Modules and standard monomial theory, Joural of the American Mathematical Society 11(1998), 551-567.

Zhixiang $\mathrm{Wu}$

Department of Mathematics

Zhejiang University

Hangzhou, 310027

P. R. China

Email: wzx@zju.edu.cn 